\title{
Elastic waves in a prestressed Mooney material
}

\section{J.A. Belward}

\begin{abstract}
The dynamic response of a prestressed incompressible Mooney material is studied by investigating plane wave propagation and the response of the material to impulsive lines of force. The choice of an initial deformation which is axially symmetric gives a particularly simple form for the secular equation for the plane wavefront velocities. The speeds of propagation and the amplitudes of the two permissible transverse waves are found and necessary and sufficient conditions for there to exist two real wave speeds in all directions are established. The simple form of the secular equation enables the response of the material to concentrated disturbances to be readily solved using Fourier transforms. The motions caused by a line of impulsive forces is examined in some detail.
\end{abstract}

\section{Introduction}

In this paper an account will be given of some investigations into the dynamic properties of a prestressed incompressible elastic material.

In previous work the propagation of finite amplitude waves in incompressible materials has been investigated by Ericksen [1] and Manacorda [6] (see also Truesdell and Noll [7]) and Knowles [5]. Flavin and Green [2] have examined the propagation of small amplitude thermoelastic waves superposed on a finite deformation for both

Received 28 March 1972. The author thanks the governing body of the School of Mathematics and Physics at the University of East Anglia for generous assistance towards a visit in the course of which this work was carried out. 
compressible and incompressible materials. The present work focusses on the particular case of an incompressible Mooney material which is subjected to an initial pure homogeneous deformation with equal extension ratios in two perpendicular directions. The propagation of plane waves in the material is discussed and the response of the material to impulsive line forces is examined in detail.

Because the material is incompressible only two plane waves are possible and these must both be transverse waves. The simple form of the secular equation which determines the wave speeds in the present problem enables the speeds of propagation to be expressed as simple functions of the material constants in the problem. The amplitudes associated with each wave are found and necessary and sufficient conditions for the propagation of plane waves in every direction of the material are given.

Fundamental problems involving the response of the material to line disturbances are examined using transform techniques. When the secular equation has the simple form which it has in the current problem the response of the material to impulsive disturbances or sustained harmonic oscillations can be reduced to simple Fourier inversions ${ }^{2}$.

A detailed analysis is made of the disturbances caused by line concentrations of forces, one acting along the axis of symmetry of the initial deformation and the other perpendicular to and passing through the axis of symmetry. In the former case the solutions are found in terms of simple algebraic functions. In the second case certain of the solutions can only be expressed as double integrals. However these solutions are shown to have the same asymptotic behaviour as the first set for $t$ small and for $t$ large. In both cases for large times the displacements are independent of position to first order. They are proportional to $t^{-1}$ and the displacement in the $i$ th direction is proportional to the component of applied force in that direction only and independent of the other components.

1 The author gratefully acknowledges the assistance of $\mathrm{Dr}$ M.A. Hayes of the University of East Anglia for finding a physical problem in which this analysis is possible. 


\section{Basic equations}

The equations of motion which form the basis of the investigations will be derived first. Consider a body of homogeneous, isotropic, incompressible, perfectly elastic material with strain energy $W$ per unit volume. Let the body be subjected to a finite static deformation, under the action of surface tractions only, in which a particle at $X_{i}$ in some rectangular cartesian coordinate system moves to the point $y_{i}$ referred to the same coordinate system. Call this state $A_{1}$. For an incompressible material the Cauchy stress $\stackrel{0}{t}_{i j}$ is given by ${ }^{2}$

$$
\stackrel{0}{t}_{i j}=-p_{0} \delta_{i j}+\left(2 \frac{\partial W}{\partial I_{1}}+2 I_{1} \frac{\partial W}{\partial I_{2}}\right) \stackrel{0}{B}_{i j}-2 \frac{\partial W}{\partial \stackrel{0}{I}_{2}}{ }_{i k} \stackrel{0}{B}_{k j} \text {, }
$$

where $\stackrel{0}{B}_{i j}$ is the left Cauchy-Green strain tensor defined by

$$
\stackrel{0}{B}_{i j}=\frac{\partial y_{i}}{\partial X_{k}} \frac{\partial y_{j}}{\partial X_{k}}
$$

and $\dot{I}_{1}$ and $\stackrel{0}{I}_{2}$ are two invariants of the deformation defined by

$$
\stackrel{0}{I}_{1}=\stackrel{0}{B}_{i i} \text { and } \stackrel{0}{I}_{2}=\frac{1}{2}\left(B_{i i}^{0} B_{j j}-B_{i j} \stackrel{B}{B}_{i j}\right) \text {, }
$$

and $p_{0}$ is a hydrostatic pressure defined up to an arbitrary constant.

Since the body is in equilibrium with no body forces applied,

$$
\frac{\partial^{0} t_{i j}}{\partial y_{j}}=0
$$

Now consider a further time dependent deformation in which the particle at $y_{i}$ moves to $x_{i}$. This second deformation will be assumed small in the sense that if

$$
x_{i}-y_{i}=g u_{i},
$$

terms in $\varepsilon^{2}$ and higher powers may be neglected in comparison with terms in $\varepsilon$. Let the stress in this new configuration be denoted by

\footnotetext{
2 Kepeated suffixes. imply summation over the values $i=1,2$ and
} 3. 


$$
\stackrel{0}{t}_{i j}+\varepsilon t_{i j}
$$

then if the strain energy, pressure and strain tensor are expanded in powers of $\varepsilon$ about their values in the state $A_{1}$, thus

$$
\begin{aligned}
W & =\stackrel{0}{W}+\varepsilon W+\ldots, \\
\tilde{p} & =\stackrel{1}{p}+\varepsilon p+\ldots, \\
\tilde{B}_{i j} & =B_{i j}+\varepsilon B_{i j}+\ldots,
\end{aligned}
$$

it can be shown that

$$
\begin{aligned}
& \text { (2.8) } t_{i j}=-p \delta_{i j}+4 u_{k, Z^{B}}{ }_{k Z} \frac{\partial W}{\frac{0}{I_{2}}}{ }_{i j}^{0} \\
& +2\left(\frac{\partial W}{\partial \stackrel{0}{I}_{1}}+I_{1} \frac{\partial W}{\partial I_{2}}\right)\left(u_{i, \imath^{B}}{ }_{2 j}+u_{j, l^{B}} \stackrel{0}{i}_{i}\right)
\end{aligned}
$$

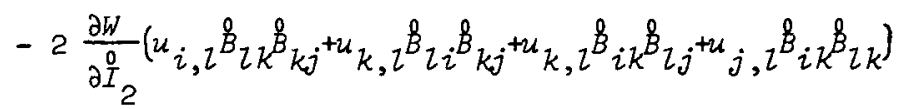

$$
\begin{aligned}
& +4 \stackrel{0}{B}_{i j}\left(J_{1} \frac{\partial}{\partial \frac{0}{I}}+J_{2} \stackrel{P}{I}_{1} \frac{\partial}{\partial I_{2}^{0}}\right)\left(\frac{\partial W}{\partial I_{1}}+\frac{\partial W}{\partial \frac{0}{I}}\right)
\end{aligned}
$$

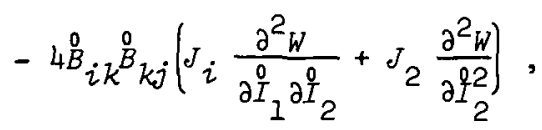

where

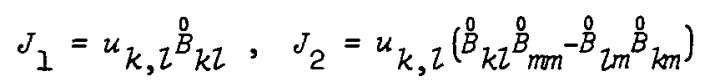

and

$$
u_{i, j}=\frac{\partial u_{i}}{\partial y_{j}}
$$

In the state $A_{2}$ the equations of motion of the body are

$$
\frac{\partial}{\partial x_{j}}\left(\stackrel{0}{t}_{i j}+\varepsilon t_{i j}\right)+\rho b_{i}=\rho \frac{\partial^{2} x_{i}}{\partial t^{2}} .
$$

The body forces which are applied to disturb the body from the state $A_{1}$ are assumed to be of the form $\varepsilon f_{i}$. Thus if equations (2.4), (2.5) 
and (2.6) are applied to equation (2.10), then to order $\varepsilon$ we have

$$
\frac{\partial t_{i j}}{\partial y_{j}}-\frac{\partial u_{k}}{\partial y_{j}} \frac{\partial t_{i j}}{\partial y_{k}}+\rho f_{i}=\rho \frac{\partial^{2} u_{i}}{\partial t^{2}} .
$$

Applying equation (2.4) reduces this equation to

$$
\frac{\partial t_{i, j}}{\partial y_{j}}+\rho f_{i}=\rho \frac{\partial^{2} u_{i}}{\partial t^{2}} .
$$

In the problems to be investigated in this paper the material of the body is assumed to be a Mooney material, and the initial deformation has principal axes of strain parallel to the coordinate axes with equal extensions in two directions. Thus we have

$$
2 W=\alpha\left(I_{1}-3\right)+\beta\left(I_{2}-3\right),
$$

and

$$
y_{1}=\mu x_{1}, y_{2}=\mu x_{2} \text {, and } y_{3}=\lambda x_{3} \text {, }
$$

with $\mu^{2} \lambda=1$, since the material is incompressible. When these expressions are used to calculate the explicit form of the perturbed stress given by (2.8) we find

$$
\begin{aligned}
& t_{11}=-p+(2 a+d) u_{1,1}+(2 b+d) u_{2,2}+d u_{3,3}, \\
& t_{12}=t_{21}=(a-b)\left(u_{2,1}+u_{1,2}\right), t_{31}=t_{13}=c u_{1,3}+a u_{3,1},
\end{aligned}
$$

$$
\begin{aligned}
& t_{22}=-p+(2 b+d) u_{1,1}+(2 a+d) u_{2,2}+d u_{3,3}, \\
& t_{32}=t_{23}=c u_{3,2}+c u_{2,3} \\
& \text { and } t_{33}=-p+d\left(u_{1,1}+u_{2,2}\right)+(2 c+d) u_{3,3}
\end{aligned}
$$

where

(2.16) $a=\mu^{2}\left(\alpha+\beta \mu^{2}\right), b=\beta \mu^{2}\left(\mu^{2}-\lambda^{2}\right), c=\lambda^{2}\left(\alpha+\beta \mu^{2}\right)$ and $d=2 \beta \mu^{2} \lambda^{2}$. Finally, since the material is incompressible we have

$$
u_{1,1}+u_{2,2}+u_{3,3}=0 \text {, }
$$

and the final form of the equations of motion (2.12) is 
(2.18) $-p, 1+20 u_{11,1}+(a-b) u_{1,22}+c u_{1,33}+(a+b) u_{2,21}$ $+\infty u_{3,31}=\rho u_{1, t t}-\rho f_{1}$,

(2.19) $-p, 2+(a+b) u_{1,21}+(a-b) u_{2,11}+2 a u_{2,22}+c u_{2,33}$ $+o u_{3,32}=\rho u_{2, t t}-\rho f_{2}$, (2.20) $-p, 3+c u_{1,31}+c u_{2,32}+a u_{3,11}+a u_{3,22}$ $+2 c u_{3,33}=\rho u_{3, t t}-\rho f_{3}$.

These are the equations of motion which will be solved in the next three sections.

\section{Elementary properties of the basic equations}

In this section we shall discuss three problems whose solutions provide important information about the fundamental dynamic properties of the material. These are the problems of propagation of plane waves, the response of the material to forced vibrations and the response to an impulsive excitation. The main results of the work concern the last of these problems which is dealt with in detail in the next two sections.

We seek plane wave solutions of equations $(2.17)-(2.20)$ by substituting

$$
\begin{aligned}
u_{k} & =A_{k}\left(y_{j}\right) \exp \left(i\left(c_{p} y_{p}-\omega t\right)\right), \\
p & =P\left(y_{j}\right) \exp \left(i\left(c_{p} y_{p}-\omega t\right)\right)
\end{aligned}
$$

into these equations and we set

$$
f_{k}=0
$$

to obtain free vibrations.

If

$$
c_{p}=S z_{p} \text {, and } \tau_{i} z_{i}=1 \text {, }
$$

then the displacements and pressure given by equations (3.1) and (3.2) represent plane waves with amplitudes $A_{k}$ and $P$ which propagate in the 
direction $z_{k}$ with velocity $(\omega / S)$. On substituting (3.1)-(3.4) into equations $(2.17)-(2.20)$ and rearranging the equations we see that $u_{k}$ and $p$ are solutions if and only if

(3.5) $i \frac{Z_{1}}{S} P+\left(2 a z_{1}^{2}+(a-b) z_{2}^{2}+c z_{3}^{2}-\rho \frac{\omega^{2}}{s^{2}}\right) A_{1}$ $+(a+b) z_{1} z_{2} A_{2}+a z_{1} z_{3} A_{3}=0$,

(3.6) $i \frac{z_{2}}{S} P+(a+b) z_{1} z_{2} A_{1}+\left((a+b) z_{1}^{2}+2 a z_{2}^{2}+c z_{3}^{2}-\rho\left(\frac{\omega^{2}}{S^{2}}\right)\right) A_{2}+a z_{2} z_{3} A_{3}=0$

(3.7) $i \frac{l_{3}}{S} p+c l_{1} z_{3} A_{1}+c z_{2} z_{3} A_{2}+\left(a z_{1}^{2}+a z_{2}^{2}+2 c z_{3}^{2}-\rho\left(\frac{\omega^{2}}{S^{2}}\right)\right) A_{3}=0$,

and

$$
i \tau_{1} A_{1}+i \tau_{2} A_{2}+i \tau_{3} A_{3}=0 \text {. }
$$

These equations are of the form

$$
M U=0,
$$

where $M$ is a $4 \times 4$ matrix and $U^{T}=\left(P, A_{1}, A_{2}, A_{3}\right)$. There exist non-trivial solutions for $U$ if and only if

$$
\operatorname{det} M=0 \text {. }
$$

Equation (3.10) is the classical secular equation. It is the equation for the permissible squared speeds of propagation in the direction $z_{k}$. In the current problem the secular equation is explicitly

$$
\left(a\left(z^{2}+z_{2}^{2}\right)+c z_{3}^{2}-\rho\left(\frac{\omega^{2}}{s^{2}}\right)\right)\left((a-b)\left(z_{1}^{2}+z_{2}^{2}\right)+c z_{3}^{2}-\rho\left(\frac{\omega^{2}}{S^{2}}\right)\right)=0 \text {. }
$$

There are thus at the most two real wave speeds in any direction in the material and since this is an equation for $\left(\frac{\omega}{S}\right)^{2}$ we require positive roots for real wave speeds. It will be stipulated that there be two real wave speeds in every direction in the material; thus from equation (3.11) we require each of $a, c$, and $(a-b)$ to be strictly positive. Recalling (2.16) we see that this implies 


$$
\mu^{2}\left(\alpha+\beta \mu^{2}\right)>0, \lambda^{2}\left(\alpha+\beta \mu^{2}\right)>0 \text { and } \mu^{2}\left(\alpha+\beta \lambda^{2}\right)>0
$$

Now $\mu^{2} \lambda=1$, so that $0<\mu, \lambda$; thus these conditions can be satisfied if and only if

$$
\alpha, \beta>0
$$

The condition (3.13) is thus the necessary and sufficient condition on the strain energy function of a Mooney material for there to be 2 waves propagated in all directions for all possible initial finite deformations of an incompressible material of the class characterised by equations $(2.14)$.

The wave speeds given by equation (3.1I) can be substituted into equations (3.5)-(3.8) to find the amplitudes. For

$$
\rho\left(\frac{\omega}{S}\right)^{2}=a\left(z_{1}^{2}+z_{2}^{2}\right)+c z_{3}^{2}
$$

the solutions are

$$
P: A_{1}: A_{2}: A_{3}=0: z_{1}: z_{2}: z_{3}-\tau_{3}^{-1} \text {, }
$$

and for

$$
\rho\left(\frac{\omega}{s}\right)^{2}=(a-b)\left(z_{1}^{2}+z_{2}^{2}\right)+c z_{3}^{2}
$$

$$
P: z_{1} A_{1}+z_{2} A_{2}: A_{3}=\frac{b z_{3}}{i}\left((a-b)+(c+b-a) z_{3}^{2}\right)^{\frac{1}{2}}:-z_{3}: 1 \text {, }
$$

where $A_{1}$ and $A_{2}$ are arbitrary within the ratios (3.17).

The problem of the response of the material to impulsive or harmonically oscillating forces can be investigated using Fourier transforms. In the initial stages no distinction need be made between the two sets of problems. We take a four-fold Fourier transform in space and time, thus:

$$
\bar{g}\left(\xi_{p}, \omega\right)=\int_{\Omega} \exp \left(i\left(y_{k} \xi_{k}+\omega t\right)\right) g\left(y_{p}, t\right) d y_{p} d t
$$

where $\Omega$ is the whole $\left(y_{p}, t\right)$ space. Equations (2.17)-(2.20) transform to the equation 
(3.19) $\quad\left(\begin{array}{cccc}-i \xi_{1} & \Xi_{1} & -(a+b) \xi_{1} \xi_{2} & -a \xi_{1} \xi_{3} \\ -i \xi_{2} & -(a+b) \xi_{1} \xi_{2} & \Xi_{2} & -a \xi_{2} \xi_{3} \\ -i \xi_{3} & -c \xi_{1} \xi_{3} & -c \xi_{2} \xi_{3} & \Xi_{3} \\ 0 & i \xi_{1} & i \xi_{2} & i \xi_{3}\end{array}\right)\left(\begin{array}{c}\bar{P}_{1} \\ \bar{u}_{1} \\ \bar{u}_{2} \\ \bar{u}_{3}\end{array}\right)=\left(\begin{array}{c}-\bar{f}_{1} \\ -\bar{f}_{2} \\ -\bar{f}_{3} \\ 0\end{array}\right)$,

that is

$$
\overline{N U}=\bar{F},
$$

where we have used the following abbreviations:

$$
\begin{aligned}
& \Xi_{1}=\left(\rho \omega^{2}-2 a \xi_{1}^{2}-(a-b) \xi_{2}^{2}-c \xi_{3}^{2}\right), \\
& \Xi_{2}=\left(\rho \omega^{2}-(a-\dot{b}) \xi_{1}^{2}-2 a \xi_{2}^{2}-c \xi_{3}^{2}\right), \\
& \Xi_{3}=\left(\rho \omega^{2}-a\left(\xi_{1}^{2}+\xi_{2}^{2}\right)-2 c \xi_{3}^{2}\right) .
\end{aligned}
$$

Thus

$$
\bar{U}=N^{-1} \bar{E}
$$

The matrix $N^{-1}$ is given by

$$
\operatorname{det} N^{-1}\left(\begin{array}{llll}
11 & 12 & 13 & 14 \\
21 & 22 & 23 & 24 \\
31 & 32 & 33 & 34 \\
41 & 42 & 43 & 44
\end{array}\right) \text {, }
$$

where 


$$
\left\{\begin{aligned}
\frac{11}{i \xi_{1}} & =\frac{12}{i \xi_{2}}=\frac{13}{i \xi_{3}}=\frac{\operatorname{det} N}{\left(\xi_{1}^{2}+\xi_{2}^{2}+\xi_{3}^{2}\right)}, \\
21 & =-\xi_{2}^{2} z_{1}-\xi_{3}^{2}\left(z_{1}-b \xi_{1}^{2}\right) \\
22 & =31=\xi_{1} \xi_{2}\left(z_{1}+b \xi_{3}^{2}\right), \\
32 & =-\xi_{1}^{2} z_{1}-\xi_{3}^{2}\left(z_{1}-b \xi_{2}^{2}\right), \\
\frac{23}{\xi_{1} \xi_{2}} & =\frac{33}{\xi_{2} \xi_{3}}=\frac{41}{\xi_{1} \xi_{2}}=\frac{42}{\xi_{2} \xi_{3}}=\frac{-43}{\xi_{1}^{2}+\xi_{2}^{2}} \\
& =z_{1}-b\left(\xi_{1}^{2}+\xi_{2}^{2}\right)
\end{aligned}\right.
$$

and

$$
z_{1}=a\left(\xi_{1}^{2}+\xi_{2}^{2}\right)+c \xi_{3}^{2}-\rho \omega^{2}
$$

Also

$$
\text { (3.22) } \operatorname{det} N=-\left(\xi_{1}^{2}+\xi_{2}^{2}+\xi_{3}^{2}\right)\left((a-b)\left(\xi_{1}^{2}+\xi_{2}^{2}\right)+c \xi_{3}^{2}-\rho \omega^{2}\right)\left(a\left(\xi_{1}^{2}+\xi_{2}^{2}\right)+c \xi_{3}^{2}-\rho \omega^{2}\right) \text {. }
$$

The last colum of $N^{-1}$ is irrelevent because the last element in $F$ is always zero. The problem is completed by finding the inverse transform of $\bar{U}$.

We distinguish between the quasi-steady problems in which

$$
u_{k}=u_{k}^{*}\left(y_{p}\right) e^{i \omega t}, p=p^{*}\left(y_{p}\right) e^{i \omega t} \text { and } f_{k}=f_{k}^{*} e^{i \omega t} \text {, }
$$

and the genuinely time dependent problems, by regarding $\omega$ as a constant in the former cases. For when equations (3.23) are introduced into equations $(2.17)-(2.20)$ and Fourier transforms taken in $y_{p}$, then equations (3.19) result. In the case of the problems of the response to an impulse the forcing functions $f_{k}$ have the form

$$
f_{k}=f_{k}\left(y_{p}\right) \delta(t) \text {, }
$$

and since the Fourier transform of $\delta(t)$ is unity it can be seen that the function $\bar{F}$ which appears in equation (3.20) does not involve $\omega$. From 
the form of the elements of $N^{-1}$ it follows that the solutions of these two groups of problems reduce to the problems of inverting functions of the form

$$
\frac{\phi\left(\xi_{k}\right)}{\left(\xi_{1}^{2}+\xi_{2}^{2}+\xi_{3}^{2}\right]\left[a\left(\xi_{1}^{2}+\xi_{2}^{2}\right)+c \xi_{3}^{2}-\rho \omega^{2}\right]\left((a-b)\left(\xi_{1}^{2}+\xi_{2}^{2}\right)+c \xi_{3}^{2}-\rho \omega^{2}\right)}
$$

Because we shall consider the response to point or line forces the function $\phi$ will always be a polynomial in the $\xi^{\prime} s$. The factorisation of the denominator is essential if the inverses of these functions are to be reduced to manageable expressions. In turn this factorisation is only possible if the secular equation has some particularly simple form as it does in this problem.

In the next sections the response of the body to an impulsive line of force will be examined in detail when the line of force is aligned along the $y_{3}$ axis and when the line of force is aligned along the $y_{1}$ axis, that is, when

$$
\begin{aligned}
& \text { (i) } f_{k}=\delta(t) \delta\left(y_{1}\right) \delta\left(y_{2}\right)\left(0, w_{2}, w_{3}\right) \text {, and } \\
& \text { (ii) } f_{k}=\delta(t) \delta\left(y_{2}\right) \delta\left(y_{3}\right)\left(w_{1}, w_{2}, w_{3}\right) \text {, } \\
& \text { (each of } w_{j} \text { is constant). }
\end{aligned}
$$

\section{An impulsive line of force along the $y_{3}$ axis}

If a line of force acts on the material with constant force per unit length and fixed orientation along the entire $y_{3}$ axis then the proilem becomes two-dimensional in the $y_{1} y_{2}$ plane. Since the initial deformation is axially symmetric with respect to the $y_{3}$ axis there is no loss of generality in assuming that the component of the force perpendicular to the $y_{3}$ axis acts in the $y_{2}$ direction. When the applied force is an impulsive force the body force terms are:

(4.1) $f_{1}=0, f_{2}=w_{2} \delta(t) \delta\left(y_{1}\right) \delta\left(y_{2}\right), f_{3}=w_{3} \delta(t) \delta\left(y_{1}\right) \delta\left(y_{2}\right)$.

These transform to give 
(4.2)

$$
\bar{f}_{1}=0, \bar{f}_{2}=w_{2} \delta\left(\xi_{3}\right), \bar{f}_{3}=w_{3} \delta\left(\xi_{3}\right)
$$

From the inverse $N^{-1}$ given by equations (3.21) and (3.22) and equation (3.20) we deduce that

$$
\bar{p}=\frac{-W_{2}{ }^{i \xi_{2}}}{\xi_{1}^{2}+\xi_{2}^{2}} \delta\left(\xi_{3}\right)
$$

$$
\bar{u}_{1}=\frac{-w_{2} \xi_{1} \xi_{2} \delta\left(\xi_{3}\right)}{\left(\xi_{1}^{2}+\xi_{2}^{2}\right]\left[(a-b)\left(\xi_{1}^{2}+\dot{\xi}_{2}^{2}\right]-\rho \omega^{2}\right]} \text {, }
$$

$$
\bar{u}_{2}=\frac{w_{2} \xi_{1}^{2} \delta\left(\xi_{3}\right)}{\left(\xi_{1}^{2}+\xi_{2}^{2}\right]\left[(a-b)\left[\xi_{1}^{2}+\xi_{2}^{2}\right]-\rho \omega^{2}\right]}
$$

and

$$
\bar{u}_{3}=\frac{w_{3} \delta\left(\xi_{3}\right)}{\left(a\left(\xi_{1}^{2}+\dot{\xi}_{2}^{2}\right)-\rho \omega^{2}\right)}
$$

The property of the delta function

$$
\delta(\alpha) f(\alpha)=\delta(\alpha) f(0)
$$

has been used repeatedly here.

The inversions of $\bar{p}$ and $\bar{u}_{3}$ are straightforward; thus since the

inverse of $\left(\xi_{1}^{2}+\xi_{2}^{2}\right)^{-1}$ with respect to $\xi_{1}$ and $\xi_{2}$ is $-(2 \pi)^{-1} \log \left(y_{1}^{2}+y_{2}^{2}\right)^{\frac{1}{2}}$ and since $i \xi_{1} \bar{f}\left(\xi_{k}\right)=\frac{\overline{\partial f}}{\partial y_{1}}$, we have

$$
p=\frac{W_{2}}{2 \pi} \frac{y_{2}}{y_{1}^{2}+y_{2}^{2}} \delta(t) .
$$

$\bar{u}_{3}$ can be inverted from first principles, or it may be observed that it is the transform of a fundamental solution of the two-dimensional wave equation. It is found that 
$(4.8)$

$$
u_{3}= \begin{cases}\frac{w_{3}}{2 \pi a}\left(t^{2}-\frac{\rho}{a}\left(y_{1}^{2}+y_{2}^{2}\right)\right]^{-\frac{1}{2}}, & t>\left[\frac{\rho\left(y_{1}^{2}+y_{2}^{2}\right)}{a}\right]^{\frac{1}{2}}, \\ 0, & t<\left[\frac{\rho\left(y_{1}^{2}+y_{2}^{2}\right)}{a}\right]^{\frac{1}{2}} .\end{cases}
$$

The inverses of $\bar{u}_{1}$ and $\bar{u}_{2}$ have to be found from the inverse of

$$
\bar{\psi}\left(\xi_{1}, \xi_{2}, \omega\right)=\left(\xi_{1}^{2}+\xi_{2}^{2}\right)^{-1}\left((a-b)\left(\xi_{1}^{2}+\xi_{2}^{2}\right)-\rho \omega^{2}\right)^{-1}
$$

Since the inverses of the two terms on the right-hand side of (4.9) are known, the inverse of $\bar{\psi}$ is just the convolution of these inverses:

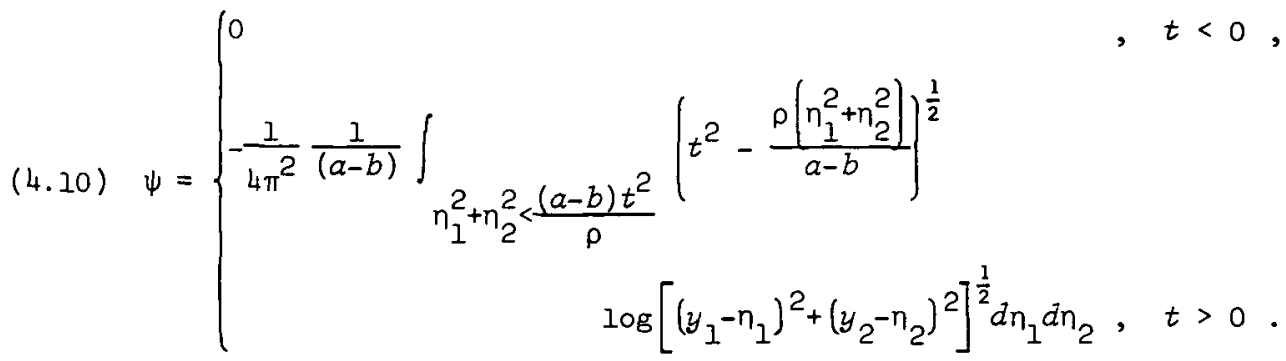

The integral can be evaluated in terms of elementary functions by changing to polar coordinates; thus for $t>0$,

$$
\begin{aligned}
& \text { (4.11) } \psi=-\frac{1}{4 \pi^{2}(a-b)} \int_{\sigma_{0}^{2}<\frac{a-b}{\rho} t}\left(t^{2}-\frac{\rho \sigma_{0}^{2}}{a-b}\right)^{-\frac{1}{2}} \\
& \log \left(\sigma^{2}+\sigma_{0}^{2}-2 \sigma \sigma_{0} \cos \left(\theta-\theta_{0}\right)\right)^{\frac{1}{2}} \sigma_{0} d \sigma_{0} d \theta_{0},
\end{aligned}
$$

(where $(\sigma, \theta)$ and $\left(\sigma_{0}, \theta_{0}\right)$ are the polar coordinates of $\left(y_{1}, y_{2}\right)$ and $\left(n_{1}, n_{2}\right)$ respectively). The integral with respect to $\theta_{0}$ can be carried out by expanding the logarithm in a power series in $\left(\frac{\sigma}{\sigma_{0}}\right)$ or $\left(\frac{\sigma_{0}}{\sigma}\right)$, whichever is smaller, and integrating term by term. We obtain 
148

J.A. Bel ward

(4.13a) $\psi\left(y_{1}, y_{2}, t\right)=$

$$
\begin{aligned}
& -\frac{1}{4 \pi^{2}(a-b)} \int_{0}^{\left(\frac{a-b}{\rho}\right)^{\frac{1}{2}} t}\left(t^{2}-\frac{\rho \sigma_{0}^{2}}{a-b}\right)^{-\frac{1}{2}} 2 \pi\left\{\begin{array}{l}
\log \sigma_{0}, \sigma_{0}>\sigma \\
\log \sigma, \sigma>\sigma_{0}
\end{array}\right\} \sigma_{0} d \sigma_{0}, \\
& \text { for } t>0 \text {. }
\end{aligned}
$$

Finally $u_{1}$ and $u_{2}$ equal $\frac{\partial^{2} \psi}{\partial y_{1} \partial y_{2}}$ and $\frac{\partial^{2} \psi}{\partial y_{1}^{2}}$ and thus

$(4.12) \quad u_{1}=\left\{\begin{array}{l}\frac{w_{2}}{\pi}\left[\frac{y_{1} y_{2}}{\left(y_{1}^{2}+y_{2}^{2}\right)^{2}} \frac{1}{\rho}\left[t-\left(t^{2}-\frac{\rho\left(y_{1}^{2}+y_{2}^{2}\right)}{a-b}\right)^{\frac{1}{2}}\right]-\right. \\ \left.\frac{y_{1} y_{2}}{2\left(y_{1}^{2}+y_{2}^{2}\right)} \frac{1}{(a-b)}\left(t^{2}-\frac{\rho\left(y_{1}^{2}+y_{2}^{2}\right)}{a-b}\right)^{-\frac{1}{2}}\right], \text { for }\left(y_{1}^{2}+y_{2}^{2}\right)^{\frac{1}{2}}<\frac{a-b}{\rho} t, \\ \frac{w_{2}}{\pi \rho} \frac{y_{1} y_{2} t}{\left(y_{1}^{2}+y_{2}^{2}\right)^{2}}, \text { for } 0<\frac{(a-b)}{\rho} t<\left(y_{1}^{2}+y_{2}^{2}\right)^{\frac{1}{2}}, \\ 0 \quad \text { for } t<0,\end{array}\right.$

and

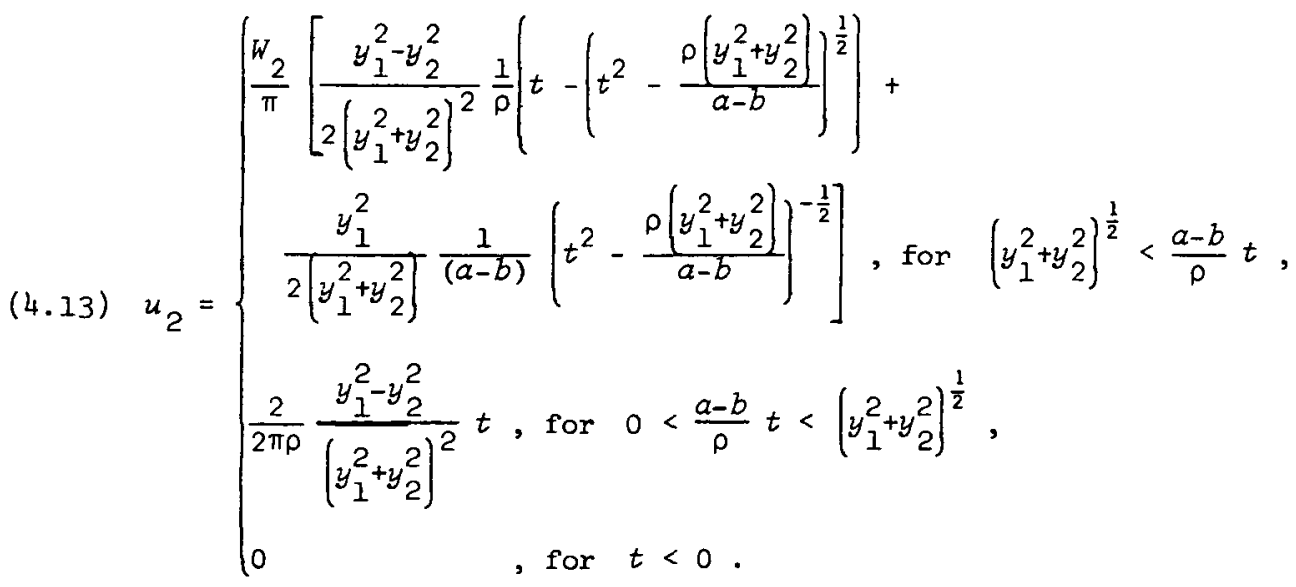

For $t^{2} \gg\left(y_{1}^{2}+y_{2}^{2}\right)$ we have

https://doi.org/10.1017/S0004972700044907 Published online by Cambridge University Press 
$(4.14)$

$$
\left\{\begin{array}{l}
u_{1} \sim \frac{w_{2} \rho}{8 \pi(a-b)^{2}} \frac{y_{1} y_{2}}{t^{3}}, \\
u_{2} \sim \frac{w_{2}}{4(a-b)} \frac{1}{t}, \\
u_{3} \sim \frac{w_{3}}{2 \pi a t},
\end{array}\right.
$$

$(p=0$ for all $t \neq 0)$.

These solutions show that there are two waves which travel independently of one another, one carrying a displacement signal in the $y_{3}$ direction, due only to the force in that direction, and one carrying displacement signals in the $y_{1}$ and $y_{2}$ directions. The former wave is the classical solution to the wave equation in two dimensions due to a point impulse. The second wave has a more complicated structure; there is an immediate response in the $y_{1}$ and $y_{2}$ directions, the displacements increase linearly in time until

$$
t=\rho^{\frac{1}{2}}(a-b)^{-\frac{1}{2}}\left(y_{1}^{2}+y_{2}^{2}\right)^{\frac{1}{2}},
$$

then they fall to zero again as $t$ increases further. For large time both $u_{2}$ and $u_{3}$ fall off as $\frac{1}{t}$, but independent of position. The displacement $u_{1}$ decays like $\frac{1}{t^{3}}$ at a rate depending on $y_{1}$ and $y_{2}$.

The presence of an imediate change in the displacements can be attributed to the incompressibility of the material which can be regarded as causing a wave which travels with infinite velocity.

In the next problem the solutions are not all expressible in such simple terms as in the current problem. However we will show that the main results from the current problem are preserved.

\section{An impulsive line of force along the $y_{1}$ axis}

When the line of force acts along an axis perpendicular to the $y_{3}$ 
axis there is no loss of generality in choosing the $y_{1}$ axis since the initial deformation is axially symetric with respect to the $y_{3}$ axis. However none of the components of the applied force may be assumed to be zero. The problem becomes two-dimensional in the planes $y_{1}=$ a constant . The body force terms are

$$
\begin{aligned}
\text { (5.1) } f_{1}=w_{1} \delta(t) \delta\left(y_{2}\right) \delta\left(y_{3}\right), f_{2}=w_{2} \delta(t) \delta\left(y_{2}\right) \delta\left(y_{3}\right) & \\
f_{3} & =w_{3} \delta(t) \delta\left(y_{2}\right) \delta\left(y_{3}\right) .
\end{aligned}
$$

From equations $(3.20),(3.21)$ and (3.22) we read off

$$
\begin{aligned}
p & =\frac{-w_{2} i \xi_{2}-w_{3} i \xi_{3}}{\left(\xi_{2}^{2}+\xi_{3}^{2}\right)} \delta\left(\xi_{1}\right), \\
\bar{u}_{1} & =\frac{w_{1} \delta\left(\xi_{1}\right)}{\left.(a-b) \xi_{2}^{2}+c \xi_{3}^{2}-\sigma \omega^{2}\right)} \delta\left(\xi_{1}\right), \\
\bar{u}_{2} & =\frac{w_{2} \xi_{3}^{2}-w_{3} \xi_{2} \xi_{3}}{\left(a \xi_{2}^{2}+c \xi_{3}^{2}-\sigma \omega^{2}\right]\left[\xi_{2}^{2}+\xi_{3}^{2}\right.} \delta\left(\xi_{1}\right),
\end{aligned}
$$

and

$$
\bar{u}_{3}=\frac{\left(-w_{2} \xi_{2} \xi_{3}+w_{2} \xi_{2}^{2}\right) \delta\left(\xi_{1}\right)}{\left(a \xi_{2}^{2}+c \xi_{3}^{2}-\rho \omega^{2}\right)\left(\xi_{2}^{2}+\xi_{3}^{2}\right)}
$$

Immediately we have

$$
p=\frac{\delta(t)}{2 \pi} \frac{w_{2} 2^{+w_{3} y_{3}}}{\left[y_{2}^{2}+y_{3}^{2}\right]},
$$

and

$$
(5.7) \quad u_{1}= \begin{cases}\frac{w_{1}}{2 \pi(a-b)^{\frac{1}{2}} c^{\frac{1}{2}}}\left(t^{2}-\frac{\rho y_{2}^{2}}{(a-b)}-\frac{\rho y_{3}^{2}}{c}\right)^{-\frac{1}{2}}, & t>\left(\frac{\rho y_{2}^{2}}{(a-b)}+\frac{\rho y_{3}^{2}}{c}\right)^{\frac{1}{2}}, \\ 0 & , \quad t<\left(\frac{\rho y_{2}^{2}}{(a-b)}+\frac{\rho y_{3}^{2}}{c}\right)^{\frac{1}{2}} .\end{cases}
$$


To invert $\bar{u}_{2}$ and $\bar{u}_{3}$ we require $\frac{\partial^{2} x}{\partial y_{2}^{2}}, \frac{\partial^{2} x}{\partial y_{3}^{2}}$, and $\frac{\partial^{2} x}{\partial y_{2} \partial y_{3}}$, where

$$
\bar{x}=\left(a \xi_{2}^{2}+c \xi_{3}^{2}-\rho \omega^{2}\right)^{-1}\left(\xi_{2}^{2}+\xi_{3}^{2}\right)^{-1}
$$

We may use the convolution theorem as in the previous problem; then

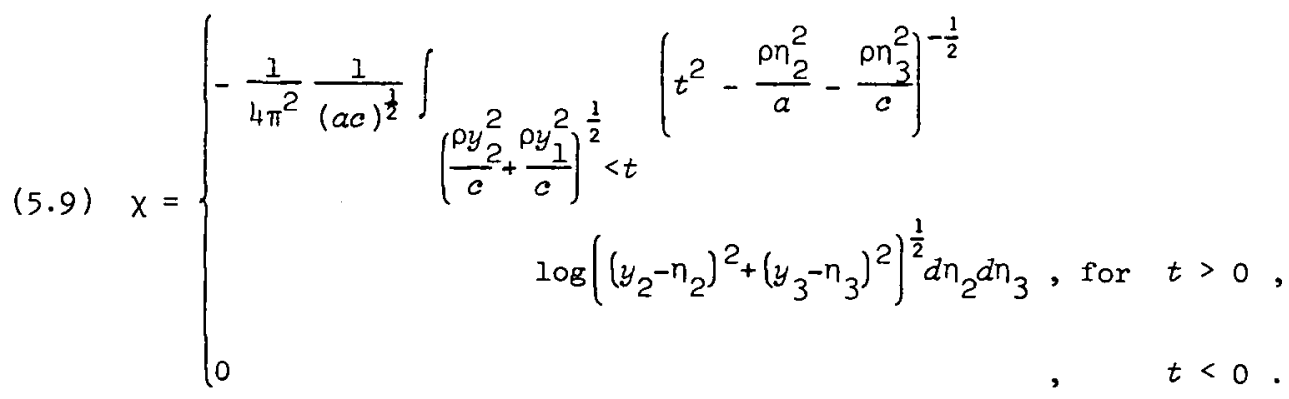

Although this integral only differs slightly from that for $\psi$, it appears that it cannot be evaluated in terms of elementary functions. The properties of the displacements $u_{2}$ and $u_{3}$ thus have to be established less directly, and because they are linear combinations of second order derivatives of $X$ the deduction of their properties is non-trivial. [Both differentiations cannot be carried under the integral sign since this gives rise to divergent integrals. Similarly, methods based on integration by parts also give divergent integrals.]

We shall find the asymptotic forms of $u_{2}$ and $u_{3}$ for $t \ll\left(y_{1}^{2}+y_{2}^{2}\right)^{\frac{1}{2}}$ and $t \gg\left(y_{1}^{2}+y_{2}^{2}\right)^{\frac{1}{2}}$, that is, at large distances in front of and behind the wavefront $t^{2}=\frac{\rho y_{2}^{2}}{a}+\frac{\rho y_{3}^{2}}{c}$. First we may note that since the integral in (5.9) can be evaluated when $c=a$, we can expand $x$ in a power series in $\frac{c-a}{a}, \frac{c-a}{c}$, according to whether $c<a$ or $c>a$. In principle $X$ and its derivatives can then be found to any accuracy for all the integrals in the series can be evaluated. However, while the first one or two terms can be calculated without much difficulty, later manipulations involve enormous calculations. 
The expansion of $x$, and thus $u_{2}$ and $u_{3}$, for small values of $t$ is straightforward. When $t^{2} \ll \frac{\rho}{a} y_{2}^{2}+\frac{\rho}{c} y_{3}^{2}$, the expression $\left(y_{2}-n_{2}\right)^{2}+\left(y_{3}-n_{3}\right)^{2}$ is never zero inside the ellipse $t^{2}=\frac{\rho}{a} y_{2}^{2}+\frac{\rho}{c} y_{3}^{2}$ and so the differentiations can be taken under the integral sign. For example

$$
\begin{aligned}
& \text { (5.10) } \left.\frac{\partial^{2} x}{\partial y_{2} \partial y_{3}}=\frac{1}{4 \pi^{2}(a c)^{\frac{1}{2}}} \int_{\left(\frac{\rho_{\eta}}{a_{2}}+\rho_{c} \eta_{3}^{2}\right.}\right)<t^{2}\left(t^{2}-\frac{\rho \eta_{2}^{2}}{a}-\frac{\rho \eta_{1}^{2}}{c}\right)^{-\frac{1}{2}} \\
& \frac{2\left(y_{2}-n_{2}\right)\left(y_{3}-n_{3}\right)}{\left(\left(y_{2}-n_{2}\right)^{2}+\left(y_{3}-n_{3}\right)^{2}\right)^{2}} d n_{2} d n_{3} .
\end{aligned}
$$

Now with $\zeta_{2}=n_{2} t$ and $\zeta_{3}=n_{3} t$ in the integral we obtain

$$
\begin{aligned}
\frac{\partial^{2} x}{\partial y_{2} \partial y_{3}}=\frac{t}{2 \pi^{2}(a c)^{\frac{1}{2}}} \int_{\frac{\rho}{a} \zeta_{2}^{2}+\frac{\rho}{c} \zeta_{3}^{2}<1}\left(1-\frac{\rho}{a} \zeta_{2}^{2}-\frac{\rho}{c} \zeta_{3}^{2}\right)^{-\frac{1}{2}} & \frac{\left(y_{2}-\zeta_{2}\right)\left(y_{3}-\zeta_{3}\right)}{\left|\left(y_{2}-\zeta_{2}\right)^{2}+\left(y_{3}-\zeta_{3}\right)^{2}\right|^{2}} d \zeta_{2} d \zeta_{3} .
\end{aligned}
$$

We may legitimately write

$$
\frac{\left(y_{2}-t \zeta_{2}\right)\left(y_{3}-t \zeta_{3}\right)}{\left(\left(y_{2}-t \zeta_{2}\right)^{2}+\left(y_{3}-t \zeta_{3}\right)^{2}\right)^{2}}=\frac{y_{2} y_{3}}{\left(y_{2}^{2}+y_{3}^{2}\right)^{2}}+o(t),
$$

thus

$$
\text { (5.11) } \begin{aligned}
\frac{\partial^{2} x}{\partial y_{2} \partial y_{3}} & =\frac{t}{2 \pi^{2}(a c)^{\frac{1}{2}}} \frac{y_{2} y_{3}}{\left(y_{2}^{2}+y_{3}^{2}\right)^{2}} \int_{\frac{\rho}{a} \zeta_{2}^{2}+\frac{\rho}{c} \zeta_{3}^{2}<1} \quad\left(1-\frac{\rho}{a} \zeta_{2}^{2}-\frac{\rho}{c} \zeta_{3}^{2}\right)^{-\frac{1}{2}} d \zeta_{2} d \zeta_{3}+o\left(t^{2}\right) \\
& =\frac{t}{\pi \rho} \frac{y_{2} y_{3}}{\left(y_{2}^{2}+y_{3}^{2}\right)^{2}}+o\left(t^{2}\right) .
\end{aligned}
$$


The expansions of the other second order derivatives of $X$ follow in like manner and thus it follows that:

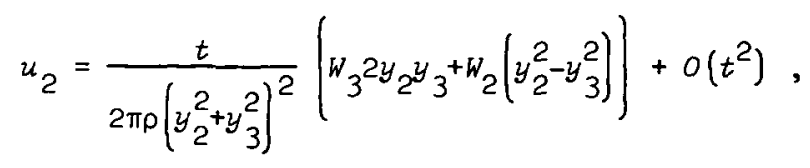

and

$$
u_{3}=\frac{t}{2 \pi \rho\left(y_{2}^{2}+y_{3}^{2}\right)^{2}}\left(w_{2} 2 y_{2} y_{3}-w_{3}\left(y_{2}^{2}-y_{3}^{2}\right)\right)+o\left(t^{2}\right)
$$

There is obviously no great difficulty in finding higher order approximations. Exact upper and lower bounds on $u_{2}$ and $u_{3}$ can also be calculated when $\frac{\rho y_{2}^{2}}{a}+\frac{\rho y_{3}^{2}}{c}>t^{2}$. These can be found from simple estimates of the integral on the right-hand side of equation (5.10) and its two. counterparts.

When $t^{2} \gg \frac{\rho}{a} y_{2}^{2}+\frac{\rho}{c} y_{3}^{2}$ the problem is more complicated. One differentiation can be taken under the integral sign and this proves to be sufficient to enable the asymptotic forms of $u_{2}$ and $u_{3}$ to be determined. First we change the variables in $X$. Let

$(5.14) \quad y_{2}=\left(\frac{\rho}{a}\right)^{\frac{1}{2}} y_{2}, \quad y_{3}=\left(\frac{\rho}{c}\right)^{\frac{1}{2}} y_{3}, \quad z_{2}=\left(\frac{\rho}{a}\right)^{\frac{1}{2}} n_{2} \quad$ and $z_{3}=\left(\frac{\rho}{a}\right)^{\frac{1}{2}} n_{3}$; then

$$
\begin{aligned}
& \text { (5.15) } x=-\frac{1}{4 \pi^{2} \rho} \int_{z_{2}^{2}+z_{3}^{2}<t^{2}}\left(t^{2}-z_{2}^{2}-z_{3}^{2}\right)^{-\frac{1}{2}} \\
& \log \left(\frac{a}{\rho}\left(y_{2}-z_{2}\right)^{2}+\frac{c}{\rho}\left(y_{3}-z_{3}\right)^{2}\right)^{\frac{1}{2}} d z_{2} d z_{3} .
\end{aligned}
$$

We denote the region $z_{2}^{2}+z_{3}^{2}<t^{2}$ by $D_{1}$, and the region $z_{2}^{2}+z_{3}^{2}<y_{2}^{2}+y_{3}^{3}$ by $D_{2}$.

It will be shown that 


$$
\text { (5.16) } \begin{aligned}
\frac{\partial x}{\partial y_{2}} & = \\
& \frac{-1}{4 \pi^{2} \rho} \int_{D_{2}}\left(t^{2}-z_{2}^{2}-z_{3}^{2}\right)^{-\frac{1}{2}} \frac{\partial}{\partial Y_{2}}\left(\log \left|\frac{a}{\rho}\left(y_{2}-z_{2}\right)^{2}+\frac{c}{\rho}\left(y_{3}-z_{3}\right)^{2}\right|\right) d z_{2} d z_{3}
\end{aligned}
$$

then the asymptotic form of $\frac{\partial X}{\partial Y_{2}}$ follows immediately. The proof of this assertion is given in Section 7 .

The asymptotic form of $\frac{\partial X}{\partial Y_{2}}$ is easily found because the domain $D_{2}$, unlike $D_{1}$, is independent of $t$. Thus we can expand $\left(t^{2}-z_{2}^{2}-z_{3}^{2}\right)^{-\frac{1}{2}}$ in inverse powers of $t$. Then

$$
\text { (5.17) } \frac{\partial x}{\partial Y_{2}} \sim-\frac{1}{4 \pi^{2} \rho} \frac{1}{t} \int_{D_{2}} \frac{\left(Y_{2}-z_{2}\right)}{a\left(y_{2}-z_{2}\right)^{2}+c\left(Y_{3}-z_{3}\right)^{2}} d z_{2} d z_{3}+o\left(\frac{1}{t^{2}}\right) \text {. }
$$

On changing the origin of integration to the point $\left(Y_{2}, Y_{3}\right)$ and changing to polar coordinates the integral becomes

$$
\int_{0<R_{0}<R \cos \left(\theta_{0}-\theta+\pi\right)}\left(\frac{-a \cos \theta_{0}}{a \cos ^{2} \theta_{0}+b \sin ^{2} \theta_{0}}\right) d R_{0} d \theta_{0}
$$

which equals

$$
\frac{2 \pi a^{\frac{1}{2}} R \cos \theta}{a^{\frac{1}{2}}+c^{\frac{1}{2}}}
$$

Thus

$$
\frac{\partial X}{\partial Y_{2}} \sim-\frac{1}{2 \pi \rho} \frac{\sqrt{a}}{\sqrt{a}+\sqrt{a}} \frac{Y_{2}}{t}
$$

Similarly we can prove that

$$
\frac{\partial X}{\partial Y_{3}} \sim-\frac{1}{2 \pi \rho} \frac{\sqrt{a}}{\sqrt{a}+\sqrt{c}} \frac{Y_{3}}{t} .
$$

The asymptotic forms of $u_{2}$ and $u_{3}$ follow from (5.18) and (5.19) and the above results, they are 


$$
u_{2} \sim \frac{1}{2 \pi} \frac{W_{2}}{(\sqrt{a}+\sqrt{c}) \sqrt{a}} \frac{1}{t} \text {, }
$$

and

$$
u_{3} \sim \frac{1}{2 \pi} \frac{w_{3}}{\sqrt{c}(\sqrt{a}+\sqrt{c})} \frac{1}{t} .
$$

Thus to the first order of approximation the displacements are independent of position at large times and the displacement in the $y_{i}$ direction is proportional only to the force in that direction.

\section{Energy flux}

Finally the energy flux vector $E_{i}$ cen be calculated for the problems of this section. This is defined to be $-\tilde{t}_{i j} V_{j}$ where $\tilde{t}_{i j}$ is the total stress and $V_{j}$ is the particle velocity. It should be noted that $\tilde{t}_{i j} V_{j}$ is in fact the flux per unit area in the state $A_{2}$. Since the displacements corresponding to the change from the state $A_{1}$ to the state $A_{2}$ are of order $\varepsilon$, to first order the energy flux per unit area can be regarded as measured in either state.

Also since

$$
\tilde{t}_{i j}=t_{i j}+\varepsilon t_{i j}
$$

we have

$$
E_{i}=-\tilde{t}_{i j} v_{j}=-t_{i j} v_{j}+O(\varepsilon) \text {. }
$$

Lastly, since the displacements calculated in Sections 4 and 5 are particle displacements, the particle velocities are simply the time derivatives of the displacements. Thus we have

$$
E_{i}=\stackrel{0}{t}_{i j} \frac{\partial u_{j}}{\partial t} \varepsilon+O\left(\varepsilon^{2}\right) .
$$

Now the matrix of $\stackrel{0}{t}_{i j}$ is diagonal with positive elements, namely, 


$$
\left(\begin{array}{ccc}
\mu^{2}\left(\alpha+\beta\left(\lambda^{2}+\mu^{2}\right)\right) & 0 & 0 \\
0 & \mu^{2}\left(\alpha+\beta\left(\lambda^{2}+\mu^{2}\right)\right) & 0 \\
0 & 0 & \lambda^{2}\left(\alpha+2 \beta \mu^{2}\right)
\end{array}\right) \text {. }
$$

It is then a simple matter to determine $E_{i}$, from which the following properties are easily noted:

(i) for $t$ small the energy flux is perpendicular to the axis of the line of force;

(ii) at all times the energy flux vector has a positive component along the velocity vector when the material is relaxing and in the opposite direction to the velocity vector when the material is undergoing increasing strain;

(iii) for large tịmes the energy flux vector has a positive component along the direction of the applied impulsive force.

7. Differentiation of the function $x$ of equation (5.9) under the integral sign

The assertion of equation (5.16) is that if

(7.1) $x=-\frac{1}{4 \pi^{2} \rho} \int_{D_{1}}\left(t^{2}-z_{2}^{2}-z_{3}^{2}\right)^{-\frac{1}{2}} \log \left(\frac{a}{\rho}\left(y_{2}-z_{2}\right)^{2}+\frac{c}{\rho}\left(y_{3}-z_{3}\right)^{2}\right)^{\frac{1}{2}} d z_{2} d z_{3}$, where $D_{1}$ is the set of points $\left(z_{2}, z_{3}\right)$ with $z_{2}^{2}+z_{3}^{2}<t^{2}$, then (7.2) $\frac{\partial x}{\partial y_{2}}=-\frac{1}{4 \pi^{2} \rho} \int_{D_{2}}\left(t^{2}-z_{2}^{2}-z_{3}^{2}\right)^{-\frac{1}{2}} \frac{\partial}{\partial y_{2}}\left(\log \left(\frac{a}{\rho}\left(y_{2}-z_{2}\right)^{2}+\frac{c}{\rho}\left(y_{3}-z_{3}\right)^{2}\right)\right) d z_{2} d z_{3}$, where $D_{2}$ is the set $z_{2}^{2}+z_{3}^{2}<y_{2}^{2}+y_{3}^{2}$.

To prove the assertion the function $X$ is first expanded in a series of powers of $\frac{a-a}{a}$ or $\frac{c-a}{c}$, which may then be integrated term by term. For definiteness assume $a>c$. Now $a$ and $c$ are necessarily positive so that $a>c>0$ implies $-1<\frac{c-a}{a}<0$. Also 


$$
\left.\frac{a}{\rho}\left(y_{2}-z_{2}\right)^{2}+\frac{c}{\rho}\left(y_{3}-z_{3}\right)^{2} \equiv \frac{a}{\rho}\left[\left(y_{2}-z_{2}\right)^{2}+y_{3}-z_{3}\right)^{2}\right]\left[1+\frac{c-a}{a} \frac{\left(y_{3}-z_{3}\right)^{2}}{\left(y_{2}-z_{2}\right)^{2}+\left(y_{3}-z_{3}\right)^{2}}\right]
$$

and since

$$
\left|\frac{c-a}{a} \frac{\left(y_{3}-z_{3}\right)^{2}}{\left(y_{2}-z_{2}\right)^{2}+\left(y_{3}-z_{3}\right)^{2}}\right|<1
$$

the logarithm term in (7.1) can be expanded to give

$$
\text { (7.3) } \begin{aligned}
x=-\frac{1}{4 \pi^{2} \rho} \int_{D_{1}}\left(t^{2}-z_{2}^{2}-z_{3}^{2}\right)^{-\frac{1}{2}}\left[\log \left(\frac{a}{\rho}\left[\left(y_{2}-z_{2}\right)^{2}+\left(y_{3}-z_{3}\right)^{2}\right]\right)\right. \\
+\sum_{n=1}^{\infty} \frac{(-1)^{n+1}}{n}\left(\frac{c-a}{a}\right)^{n} \frac{\left(y_{3}-z_{3}\right)^{2 n}}{\left.\left[\left(y_{2}-z_{2}\right)^{2}+\left(y_{3}-z_{3}\right)^{2}\right]^{n}\right] d z_{2} d z_{3}} .
\end{aligned}
$$

Consider the integral involved in a typical term of the series in (7.3). The integral of the first term was dealt with in Section 4 in equation (4.10-4.11a). The remaining terms are of the form

(7.4) $G_{n}\left(y_{2}, y_{3}\right)=\int_{D_{1}}\left(t^{2}-z_{2}^{2}-z_{3}^{2}\right)^{-\frac{1}{2}} \frac{\left(y_{3}-z_{3}\right)^{2 n}}{\left|\left(y_{2}-z_{2}\right)^{2}+\left(y_{3}-z_{3}\right)^{2}\right|^{n}} d z_{2} d z_{3}$.

By changing to polar coordinates:

$$
Y_{2}=R \cos \theta, Y_{3}=R \sin \theta, Z_{2}=R_{0} \cos \theta_{0}, Z_{3}=R_{0} \sin \theta_{0},
$$

and integrating first with respect to $\theta_{0}$, we obtain the integral

$$
\text { (7.5) } E_{n}\left(R, \theta ; R_{0}\right)=\int_{0}^{2 \pi} \frac{\left(R \sin \theta-R_{0} \sin \theta_{0}\right)^{2 n}}{\left|\left(R \cos \theta-R_{0} \cos \theta_{0}\right)^{2}+\left(R \sin \theta-R_{0} \sin \theta_{0}\right)^{2}\right|^{n}} d \theta_{0} \text {. }
$$

Now we observe that

(i) $\left(F_{n}\left(R, \theta ; R_{0}\right)\right.$ is continuous at $R=R_{0}$, since the integrand is bounded in a neighbourhood of $\theta_{0}=\theta$ and continuous for all other values of $R, \theta$, and $R_{0} ;$ and 
(ii) that for $R_{0}>R, F_{n}\left(R, \theta ; R_{0}\right)$ is a constant independent of $R, \theta$ and $R_{0}$.

The second property is proved as follows: By substituting $\theta_{0}=\theta_{0}^{\prime}+\theta$ and observing the periodicity of the integrand we have

$$
F_{n}\left(R, \theta ; R_{0}\right)=\int_{0}^{2 \pi} \frac{\left[R \sin \theta-R_{0} \sin \left(\theta_{0}+\theta\right)\right]^{2 n}}{\left(F^{2}+R_{0}^{2}-2 R R_{0} \cos \theta_{0}\right]^{n}} d \theta_{0},
$$

and with $\mu=\frac{R}{R_{0}}$,

$$
F_{n}\left(R, \theta ; R_{0}\right)=\int_{0}^{2 \pi} \frac{\left(\mu \sin \theta-\sin \left(\theta_{0}+\theta\right)\right)^{2 n}}{\left(1-2 \mu \cos \theta_{0}+\mu^{2}\right)^{n}} d \theta_{0} .
$$

Now the numerator can be expanded to give terms of the form $K_{p}(\theta) \mu^{2 n-2 p} \sin ^{2 p}\left(\theta+\theta_{0}\right)$, and these in turn can be expressed as sums of cosines of even multiples of $\theta_{0}$, thus:

$b_{p}(\theta) \cos 2 p \theta_{0}+b_{p-1} \cos (2 p-2) \theta_{0}+\ldots+b_{0}$. From Gradshteyn and Ryzhik ([4], $\$ 3.616,(7)$ ) for $\mu<1$, (that is, $R_{0}>R$ ), we have

$$
\int_{0}^{2 \pi} \frac{\cos 2 p \theta_{0} d \theta_{0}}{\left(1-2 \mu \cos \theta_{0}+\mu^{2}\right)^{n}}=\left(1-\mu^{2}\right)^{1-2 n}\left(\text { a polynomial of degree } n+p-1 \text { in } \mu^{2}\right)
$$

Thus for $R>R_{0}$,

$$
F_{n}\left(R, \theta ; R_{0}\right)=\frac{\text { (a polynomial of degree } \left.2 n-1 \text { in } \mu^{2}\right)}{\left(1-\mu^{2}\right)^{2 n-1}}
$$

Now $\left(1-\mu^{2}\right)^{2 n-1}$ must divide the numerator exactly in the above equation, for otherwise it implies that $F_{n}$ involves a term $\left(R^{2}-R_{0}^{2}\right)^{-1}$ which is not integrable with respect to $R_{0}$. But since the integral of $F_{n}$, with respect to $R_{0}$, gives $G_{n}$, the existence of the integral on the right-hand side of equation (7.4) guarantees the existence of the 
iterated integrals. Thus from (7.7), when $R>R_{0}, F_{n}$ can only be a function of $\theta$. Putting $\mu=0$ will determine its value. It equals

$$
\int_{0}^{2 \pi} \sin ^{2 n}\left(\theta_{0}+\theta\right) d \theta
$$

and this integral equals $\frac{(2 n-1) ! !}{2 n ! !} 2 \pi$, which is independent of $x$ also as asserted. Explicitly, for $R>R_{0}$,

$$
E_{n}\left(R, \theta ; R_{0}\right)=\frac{(2 n-1) ! !}{2 n ! !} 2 \pi
$$

(At this stage no approximations have been made for finding $x$. The exact forms of $F_{n}$ can be evaluated and the integration with respect to $\theta$ completed; all the integrals are elementary, if not concise. Thus the expansion of $\theta$, and hence of $u_{2}$ and $u_{3}$, in powers of $\frac{c-a}{a}$ can be found. In practice the calculations became overwhelmingly complicated after the term in $\left(\frac{c-a}{a}\right)^{2}$.) By summing the series expansion to recapture (7.1) again and using the property of $F_{n}$ just proved, it follows that (7.9) $\mathrm{x}=-\frac{1}{4 \pi^{2} \rho} \int_{D_{1}}\left(t^{2}-R_{0}^{2}\right)^{-\frac{1}{2}} R_{0}\left(k H\left(R_{0}-R+H\left(R-R_{0}\right) p\left(R, \theta ; R_{0}\right)\right) d R_{0}\right.$ ， where $H$ is Heaviside's step function and $F$ is a function whose only important property is that $\underset{F_{0} \rightarrow R_{-}}{\lim } F\left(R, \theta ; R_{0}\right)=k$ (the last remark follows from the continuity of each $F_{n}\left(R, \theta ; R_{0}\right)$ and the continuity of the integrand of equation (4.1la) ). This property of $F$ is crucial, for

$$
\begin{aligned}
\frac{\partial}{\partial Y_{2}}\left(k H\left(R-R_{0}\right)\right. & \left.+H\left(R_{0}-R\right) F\left(R, R_{0} ; \theta\right)\right) \\
& =\frac{Y_{2}}{R}\left(-k \delta\left(R-R_{0}\right)+\delta\left(R_{0}-R\right) F\left(R, R_{0} ; \theta\right)\right)+H\left(R-R_{0}\right) \frac{\partial}{\partial Y_{2}}\left\{F\left(R, R_{0} ; \theta\right)\right\} .
\end{aligned}
$$

But since $\delta(u)=\delta(-u)$ and $\delta(u) \phi(u)=\delta(u) \phi(0)$ at a point of continuity of $\phi$, it follows that 
(7.10) $\frac{\partial}{\partial Y_{2}}\left(k H\left(R-R_{0}\right)+H\left(R-R_{0}\right) F\left(R, R_{0} ; \theta\right)\right)=H\left(R-R_{0}\right) \frac{\partial}{\partial Y_{2}} F\left(R, R_{0} ; \theta\right)$.

Finally, from equation $(5.24)$ we obtain

$$
\frac{\partial x}{\partial Y_{2}}=-\frac{1}{4 \pi^{2} \rho} \int_{D_{2}}\left(t^{2}-Z_{2}^{2}-Z_{3}^{2}\right)^{-\frac{1}{2}} \frac{\partial}{\partial Y_{2}}\left(\log \left|\frac{a}{\rho}\left(Y_{2}-Z_{2}\right)^{2}+\frac{c}{\rho}\left(Y_{3}-Z_{3}\right)^{2}\right|^{\frac{1}{2}}\right) d Z_{2} d Z_{3},
$$

the assertion of equation (7.2).

\section{References}

[1] J.L. Ericksen, "On the propagation of waves in isotropic incompressible perfectly elastic materials", J. Rational Mech. Anal. 2 (1953), 329-337.

[2] J.N..Flavin, A.E. Green, "Plane thermo-elastic waves in an initially stressed medium", J. Mech. Phys. Solids 9 (1961), 179-190.

[3] I.M. Gelfand and G.E. Shilov, Generalized functions, Vol. 1

(translated by Eugene Saletan. Academic Press, New York, London, $1964)$.

[4] I.S. Gradshteyn, I.M. Ryzhik, Table of integrals, series, and producte, 4th ed. (translated by Scripta Technica, Inc. Academic Press, New York, London, 1965).

[5] James K. Knowles, "Large amplitude oscillations of a tube of incompressible elastic material", Quart. Appl. Math. 18 (1960), $71-77$.

[6] T. Manacorda, "Sulla propagazione di onde ordinarie di discontinuità nella elasticità di secondo grado per solidi incomprimibili", Riv. Mat. Univ. Parma 10 (1959), 19-33.

[7] C. Truesdell, W. Noll, "The non-linear field theories of mechanics", Handbuch der Physik, Band III/3 (Springer-Verlag, Berlin, Hei delberg, New York, 1965).

University of Queensland,

St Lucia,

Queensland. 